Published in final edited form as:

J Am Chem Soc. 2008 January 23; 130(3): 818-820.

\title{
A Single-Molecule Nanopore Device Detects DNA Polymerase Activity With Single-Nucleotide Resolution
}

\author{
Scott L. Cockroft ${ }^{\dagger}$, John Chu ${ }^{\dagger}$, Manuel Amorin $\dagger$, Hagan Bayley $\ddagger$, and M. Reza Ghadiri ${ }^{\dagger}$ \\ $\uparrow$ Departments of Chemistry and Molecular Biology and The Skaggs Institute for Chemical Biology, The \\ Scripps Research Institute, 10550 North Torrey Pines Road, La Jolla, CA 92037, USA \\ $\ddagger$ Department of Chemistry, University of Oxford, Chemistry Research Laboratory, Mansfield Road, Oxford, \\ OXI 3TA, UK.
}

\begin{abstract}
The ability to monitor DNA polymerase activity with single-nucleotide resolution has been the cornerstone of a number of advanced single-molecule DNA sequencing concepts. Toward this goal, we report the first spatially-resolved observation of DNA polymerase activity with single-base resolution at the single-molecule level. We describe the design and characterization of a singlespecies supramolecular nanopore device capable of detecting up to nine consecutive DNA polymerase-catalyzed single nucleotide primer extensions with high sensitivity and spatial resolution $(\leq 2.4 \AA)$. The device is assembled in a suspended lipid membrane by threading and mechanically capturing a single strand of DNA-PEG copolymer inside an $\alpha$-hemolysin protein pore. Single nucleotide primer extensions result in successive displacements of the template DNA strand within the protein pore, which can be monitored by the corresponding stepped changes in the ion current flowing through the pore under an applied transmembrane potential. The system described thus represents a promising advance toward nanopore-mediated single-molecule DNA sequencing concept, and in addition might be applicable to studying a number of other biopolymer-protein interactions and dynamics.
\end{abstract}

The ability to monitor DNA polymerase activity with single-nucleotide resolution is the cornerstone of a number of advanced single-molecule DNA sequencing concepts. ${ }^{1}$ While stateof-the-art single-molecule studies of oligonucleotide-protein complexes have achieved astonishing sub-nanometer resolutions, the base-by-base activity of DNA polymerase has not been previously resolved. ${ }^{2}$ Here we describe a single-species supramolecular nanopore device capable of detecting up to nine consecutive DNA polymerase-catalyzed single nucleotide primer extensions with high sensitivity and spatial resolution $(\leq 2.4 \AA)$. The device is assembled in a suspended lipid membrane by threading and mechanically capturing a single strand of DNA-PEG copolymer inside an $\alpha$-hemolysin protein pore. ${ }^{3}$ Single nucleotide primer extensions result in successive displacements of the template DNA strand within the protein pore, which can be monitored by the corresponding stepped changes in the ion current flowing through the pore under an applied transmembrane potential. Since DNA primer extension can be observed with single-base resolution at the single-molecule level, the approach provides a plausible handle for the extraction of sequence information from individual oligonucleotide molecules. The system described represents a promising advance toward nanopore-mediated single-molecule DNA sequencing, ${ }_{5}^{4}$ and is expected to be useful for studying other biopolymerprotein interactions and dynamics. 5

E-mail: ghadiri@scripps.edu.

Supporting Information Available: Materials, Methods and Figures S1 to S17. 
Single-molecule methods have provided unique views of biomolecules in action. ${ }^{2,6}$ Amongst the emerging single-molecule techniques, Staphylococcus aureus $\alpha$-hemolysin $(\alpha-\mathrm{HL})$ has been employed as the sensor element to probe a range of molecular systems and processes ${ }^{3-}$ 5 . $\alpha$-HL forms a mushroom-shaped heptameric protein pore in lipid bilayers with a central channel of approximately $10 \mathrm{~nm}$ in length and $1.5 \mathrm{~nm}$ in diameter at its narrowest point. ${ }^{7}$ The pore dimensions can accommodate the entry and passage of a variety of small molecules and linear polymers including single-stranded DNA (ssDNA) and polyethylene glycols (PEG). ${ }^{3-}$ 5 A single $\alpha$-HL protein can be readily inserted into an artificial planar lipid membrane. Due to its constant open pore structure, a steady and characteristic ionic current results under an applied transmembrane potential. The entry of molecules into the pore gives rise to transient current modulations or blockades that can be use to characterize the molecular species involved.

We recently described a single-molecule method for threading and mechanically capturing ssDNA-PEG hybrid molecules inside the $\alpha$-HL protein pore. ${ }^{3 \mathrm{a}, \mathrm{d}}$ In the present study we have employed a similar strategy to configure a supramolecular nanopore device capable of resolving the small spatial changes associated with DNA polymerase-catalyzed single nucleotide DNA primer extensions. We reasoned that with an appropriately configured nanopore device, each single nucleotide primer extension should successively change the position of the threading strand and alter the proportion of the DNA to PEG residing within the pore. Since we have previously shown that the transmembrane ion conductance depends on whether ssDNA or PEG is held inside the pore, ${ }^{3 a}$ primer extensions were expected to give rise to stepped changes in the ion conductance that can be used to ascertain the polymerized state of the primer strand. To test this hypothesis, we employed the following thread design and nanopore device configuration.

The threading component of the nanopore device consists of a 31-nucleotide ssDNA segment bearing the primer binding sites and the template sequence, an 11-nucleotide poly $\mathrm{d}(\mathrm{A})$ section, a PEG portion made from eight units of hexaethylene glycol phosphate, and a terminal biotinyl group (Fig. 1). The design provides DNA and PEG regions of appropriate length to allow full threading of the $\alpha$-HL pore as well as the means for locking the threading strand at both ends after its insertion into the pore. Binding of streptavidin to the terminal biotin moiety creates a cap structure that is wider than the $\alpha$-HL pore entrance and provides a mechanism to direct the formation of the desired $\alpha$-HL $\cdot$ DNA-PEG configuration. ${ }^{3}$ Under the influence of an electric field the capped DNA-PEG strand can only thread through pore from its free 3'-ssDNA terminus, and can be held in that configuration as long as the applied electric field is maintained (Fig. 1a). Threading of the DNA-PEG strand is signalled by the characteristic reduction in ion conductance caused by the presence of the PEG segment inside the pore. Furthermore, since double-stranded DNA (dsDNA) is also too large to pass through the narrowest part of the $\alpha$ HL pore, a stable mechanically interlocked complex can be formed by the hybridization of a DNA primer to the 3'-ssDNA end of the threading strand protruding from the pore (Fig. 1b). Once the fully interlocked complex has been formed, the position of the threading strand inside the pore can be flipped back and forth by changing the sign of the applied transmembrane potential (Fig. 1b and 1c).

Upon application of a positive transmembrane potential, the primer-template DNA duplex region is forced into the pore and held up against the narrowest part of the channel (Fig. 1c). Therefore, according our hypothesis, changes in length of the primer were expected to afford different conductance states at positive potentials by altering the ratio of DNA to PEG occupying the channel. We tested this hypothesis by preparing a series of interlocked complexes a-d (Fig. 2a-d) using the DNA-PEG threading strand (Fig. 1d) and a set of fully complementary DNA primers ranging from 23 to 31 nucleotides in length (primers $+\mathbf{0}$ to $+\boldsymbol{8}$ ). In agreement with our hypothesis, each complex displayed a characteristic current-voltage (I$V)$ trace that depended on the length of the primer (Fig. 2e). To further establish that a change 
in the primer length could be monitored by changes in the ion conductance, we performed the following experiment. Under negative transmembrane potentials, an 8 nucleotide stretch of ssDNA in complex a resides outside the pore and is thus free to serve as an initial binding site (toe-hold) for the longer primer $(\mathbf{+ 8})$. Once bound to the toe-hold, primer $+\mathbf{8}$ should rapidly invade the adjacent duplex region and replace the shorter primer $(+\mathbf{0})$ via a three-way branch migration mechanism (Fig. 3g experiment D, and S3). ${ }^{8}$ Indeed, addition of primer $+\mathbf{8}$ to complex a led to an almost immediate and irreversible change in the ion conductance to a state corresponding to that of complex d (Fig. S15). These initial experiments established that the ion current readout provides a direct measure of the primer length and thus give credence to the application of the nanopore device for monitoring DNA polymerase-catalyzed primer extensions.

Using the nanopore device, we opted to monitor the progress of DNA polymerase catalyzed primer elongation at $+40 \mathrm{mV}$ (monitoring mode Fig. 3a), since the largest and most consistent differences between the ion currents of the primer-template complexes were observed at this potential (Fig. 2e). Elongation of the primer is inhibited in the monitoring mode since the terminal 3'-OH of the primer, which serves as the site for DNA polymerase-catalyzed incorporation of deoxynucleotide triphosphates (dNTPs), is concealed within the pore vestibule. Conversely, primer elongation is allowed in the elongation mode when the 3'-OH of the primer strand is expelled from the pore vestibule under the applied negative transmembrane potential ( $-30 \mathrm{mV}$, Fig. 3b). No current change was observed in the monitoring mode readings when TopoTaq DNA polymerase was added to a preformed complex containing the starting primer $(\mathbf{+ 0})$. However, after the further addition of dCTP, a small increase in the average current at $+40 \mathrm{mV}$ was observed (Fig. $3 \mathrm{f}, 0$ to $0.5 \mathrm{~h}$ ). We interpreted this change as arising from the incorporation of a single deoxycytosine opposite the first base (deoxyguanosine) in the threaded template strand (Fig. 3c-d). After the addition of dATP, five further current steps were observed, corresponding to the incorporation of five bases against the remaining deoxythymidines and deoxyguanosines in the template sequence (Fig. 3d-f, 0.5 to $1.5 \mathrm{~h}$ ). Since the next two bases in the template sequence were deoxycytosines, polymerization could be paused at this primer length (Fig. 3f, +6) by deliberately delaying the addition of dGTP. Polymerization was allowed to resume by the addition of dGTP, with two further current steps indicating that the fully extended primer $(\mathbf{+ 8})$ had been synthesized by the DNA polymerase (Fig. 3f, 1.5 to $3.5 \mathrm{~h}$ ). A total of nine distinct current levels were observed during this experiment, corresponding to the starting primer $(\mathbf{+ 0})$, the seven intermediate single nucleotide incorporations $(+\mathbf{1}$ to +7 ), and the fully extended primer $(+\mathbf{8})$ (Figs. $3 \mathrm{f}$ and $3 \mathrm{~g}$, Experiment 1$)$. Since polymerization only proceeds in the presence of dNTPs that are complementary to the template DNA, and the resulting single-base steps (and pauses) can be resolved by our method, we demonstrate that sequence information can be extracted from a single piece of template DNA held within the nanopore.

We established the reproducibility and generality of this method for the observation of DNA elongation by performing multiple single-molecule polymerization experiments using primers of different starting lengths, and two different types of DNA polymerase. Nucleotide incorporation steps were assigned from the cumulative ion current changes and knowledge of dNTPs added to each experiment. An excellent agreement between the current changes corresponding to each polymerized state was obtained after alignment using a least-squares analysis (Fig. 3g). Polymerization could be halted in the absence of a required base, or alternatively by the incorporation of a terminating dideoxynucleotide triphosphate (ddNTP), which lacks the 3'-OH group required for the addition of further nucleotides (Fig. 3g, Experiments 2, 7 and $\mathrm{K} 2$ ).

The spatial resolution of the nanopore device was assessed by assuming that the incorporation of a single nucleotide displaces the DNA held inside the pore by $3.4 \AA$ (the distance between 
stacked bases in B-form DNA). From the alignment of the polymerization data in Fig. 3g, we observed a total ion current change of $1.3 \mathrm{pA}$ arising from the incorporation of 9 bases (equivalent to a translocation of the DNA-PEG strand by $9(3.4=30.6 \AA)$. In this window the average ion current noise is $\pm 0.05 \mathrm{pA}$ (Fig. $3 \mathrm{~g}$ ). Thus the $30.6 \AA$ displacement can be effectively resolved in thirteen $0.1 \mathrm{pA}$ segments, each representing a $2.4 \AA$ step $(30.6 \AA / 13=2.4 \AA)$. The nanopore device achieves its highest spatial resolution $(1.4 \AA)$ in the region where single nucleotide incorporations result in the largest current change, (i.e. +6 to +7 , Fig. $3 \mathrm{~g}$ ). The observed high special resolution $(\leq 2.4 \AA)$ of the nanopore device places it alongside the most spatially sensitive single molecule techniques available for studying biomolecular systems. ${ }^{2}$,

In conclusion, we have developed a supramolecular assembly capable of monitoring the activity of DNA polymerase as it operates on a single strand of DNA threaded through a protein pore. To our knowledge this work represents the first spatially-resolved observation of DNA polymerase activity with single-base resolution at the single-molecule level. Furthermore, the ability to detect single-nucleotide, ångstrõm-scale changes in an oligonucleotide templateprimer complex using a nanopore opens a new avenue for the study of biopolymers and their interactions with proteins. ${ }^{5}$ The single-base resolution of our approach and the ability to control the passage of DNA in single-base steps satisfy the two minimal requirements of a nanoporebased sequencing device. ${ }^{1}$ Nevertheless, further significant advances are required before nanopore-based approaches can be employed in the de novo sequencing of long stretches of DNA. 1,10

\section{Supplementary Material}

Refer to Web version on PubMed Central for supplementary material.

\section{Acknowledgment}

We are grateful to NIH (grant HG003709) for financial support. M.A. is a MEC/Fulbright Postdoctoral Fellow.

\section{References}

1. (a) Shendure J, Mitra RD, Varma C, Church GM. Nat. Rev. Genet 2004;5:335-344. [PubMed: 15143316] (b) Bayley H. Curr. Opin. Chem. Biol 2006;10:628-637. [PubMed: 17113816]

2. (a) Maier B, Bensimon D, Croquette V. Proc. Natl. Acad. Sci. USA 2000;97:12002-12007. [PubMed: 11050232] (b) Braslavsky I, Hebert B, Kartalov E, Quake SR. Proc. Natl. Acad. Sci. USA 2003;100:3960-3964. [PubMed: 12651960] (c) Bustamante C, Bryant Z, Smith SB. Nature 2003;421:423-427. [PubMed: 12540915] (d) Abbondanzieri EA, Greenleaf WJ, Shaevitz JW, Landick R, Block SM. Nature 2005;438:460-465. [PubMed: 16284617] (e) Greenleaf WJ, Block SM. Science 2006;313:801. [PubMed: 16902131]

3. (a) Sanchez-Quesada J, Saghatelian A, Cheley S, Bayley H, Ghadiri MR. Angew. Chem. Int. Ed 2004;43:3063-3067. (b) Mathe J, Visram H, Viasnoff V, Rabin Y, Meller A. Biophys. J 2004;87:32053212. [PubMed: 15347593] (c) Nakane J, Wiggin M, Marziali A. Biophys. J 2004;87:615-621. [PubMed: 15240494] (d) Ashkenasy N, Sanchez-Quesada J, Bayley H, Ghadiri MR. Angew. Chem. Int. Ed 2005;44:1401-1404.

4. (a) Kasianowicz JJ, Brandin E, Branton D, Deamer DW. Proc. Natl. Acad. Sci. USA 1996;93:1377013773. [PubMed: 8943010] (b) Vercoutere W, Winters-Hilt S, Olsen H, Deamer DW, Haussler D, Akeson M. Nat. Biotechnol 2001;19:248-252. [PubMed: 11231558] (c) Rhee M, Burns MA. Trends Biotechnol 2006;24:580-586. [PubMed: 17055093] (d) Astier Y, Braha O, Bayley H. J. Am. Chem. Soc 2006;128:1705-1710. [PubMed: 16448145]

5. (a) Movileanu L, Howorka S, Braha O, Bayley H. Nat. Biotechnol 2000;18:1091-1095. [PubMed: 11017049] (b) Howorka S, Cheley S, Bayley H. Nat. Biotechnol 2001;19:636-639. [PubMed: 11433274] (c) Cheley S, Xie H, Bayley H. ChemBioChem 2006;7:1923-1927. [PubMed: 17068836] 
(d) Hornblower B, Coombs A, Whitaker RD, Kolomeisky A, Picone SJ, Meller A, Akeson M. Nat. Methods 2007;4:315-317. [PubMed: 17339846]

6. (a) Wuite GJ, Smith SB, Young M, Keller D, Bustamante C. Nature 2000;404:103-106. [PubMed: 10716452] (b) Shaevitz JW, Abbondanzieri EA, Landick R, Block SM. Nature 2003;426:684-687. [PubMed: 14634670] (c) Revyakin A, Ebright RH, Strick TR. Proc. Natl. Acad. Sci. USA 2004;101:4776-4780. [PubMed: 15037753] (d) Revyakin A, Liu C, Ebright RH, Strick TR. Science 2006;314:1139-1143. [PubMed: 17110577] (e) Kapanidis AN, Margeat E, Ho SO, Kortkhonjia E, Weiss S, Ebright RH. Science 2006;314:1144-1147. [PubMed: 17110578] (f) Zlatanova J, van Holde K. Mol. Cell 2006;24:317-329. [PubMed: 17081984] (g) Luo G, Wang M, Konigsberg WH, Xie XS. Proc. Natl. Acad. Sci. USA 2007;104:12610-12615. [PubMed: 17640918]

7. Song L, Hobaugh MR, Shustak C, Cheley S, Bayley H, Gouaux JE. Science 1996;274:1859-1866. [PubMed: 8943190]

8. Li Q, Luan G, Guo Q, Liang J. Nucleic Acids Res 2002;30:e5. [PubMed: 11788731]

9. Moffitt JR, Chemla YR, Izhaky D, Bustamante C. Proc. Natl. Acad. Sci. USA 2006;103:9006-9011. [PubMed: 16751267]

10. (a) Shendure J, Porreca GJ, Reppas NB, Lin X, McCutcheon JR, Rosenbaum AM, Wang MD, Zhang K, Mitra RD, Church GM. Science 2005;309:1728-1732. [PubMed: 16081699] (b) Margulies M, et al. Nature 2005;437:376-380. [PubMed: 16056220] 

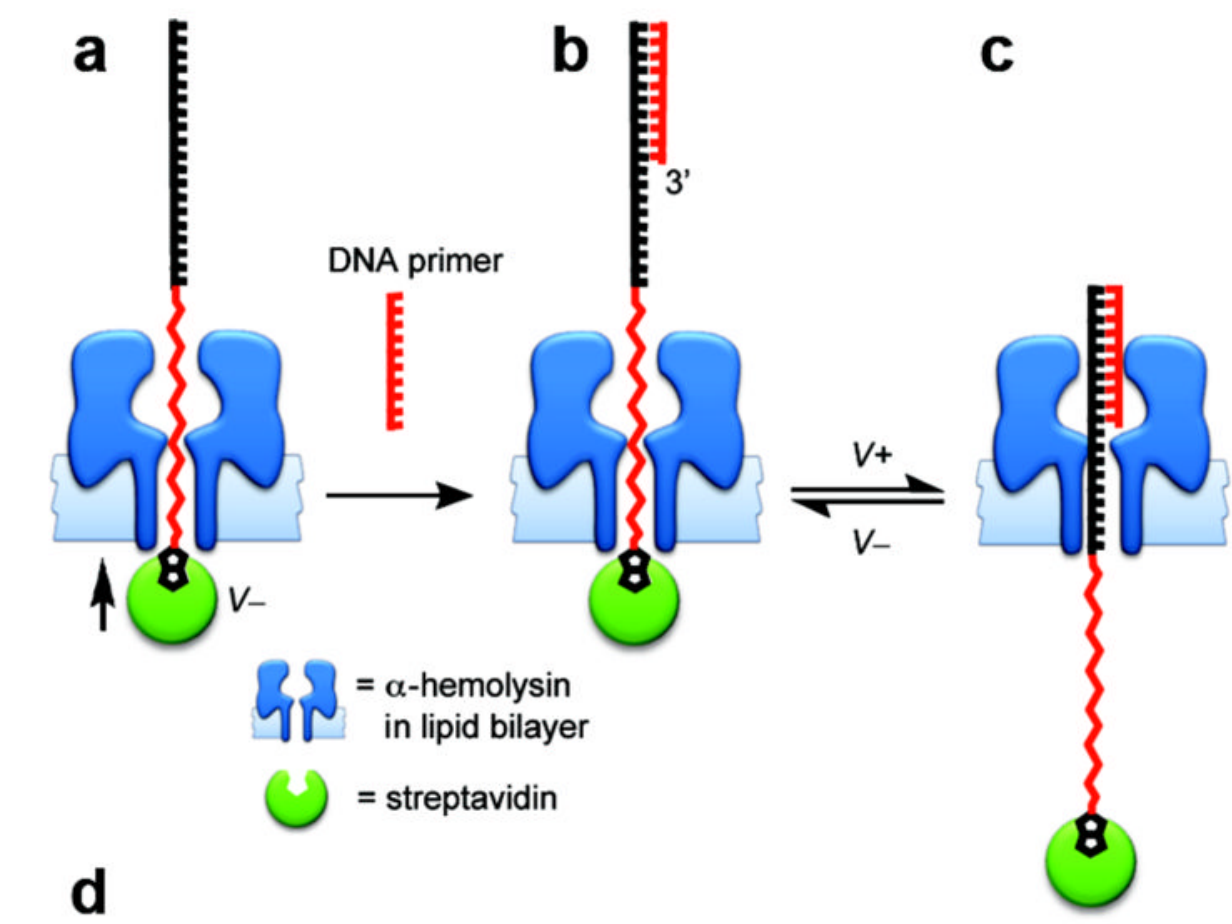

primer $(+\mathbf{0})=$ 5'-TAATACGACTGACTGTAGGGAAG-3'

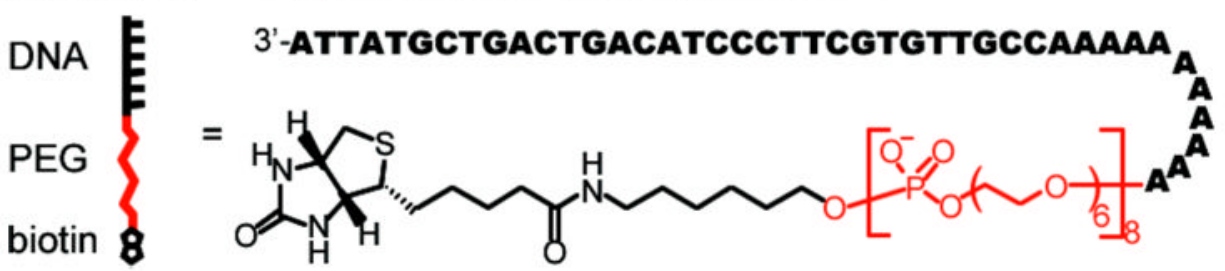

Figure 1.

Fabrication and configuration of the interlocked $\alpha-\mathrm{HL} \cdot \mathrm{DNA}-\mathrm{PEG}$ transmembrane complex. (a) A single streptavidin-capped DNA-PEG strand on one side of the membrane is driven into, and held within an $\alpha$-HL pore under an applied negative transmembrane potential. (b) Hybridization of a DNA primer to the protruding 5'-ssDNA region of the threading strand on the opposite side of the membrane, furnishes the fully interlocked $\alpha$-HL-DNA-PEG complex. (c) The position of the threading strand held inside the pore can be flipped back and forth by changing the sign of the applied potential. (d) The composition of the threading DNA-PEG strand. 

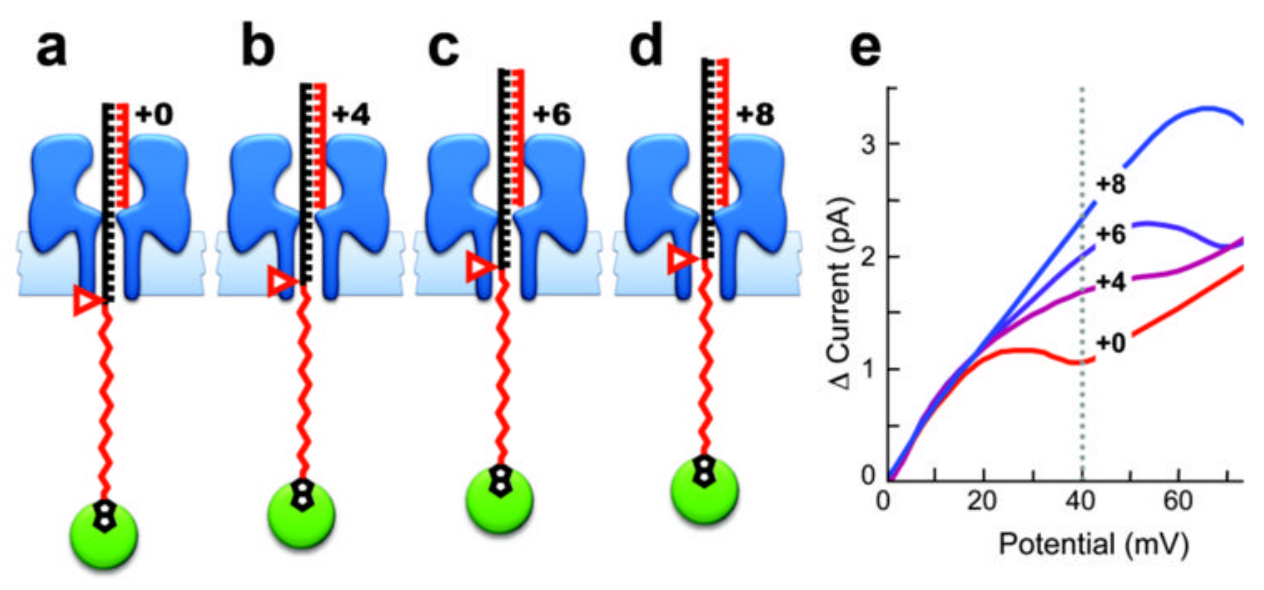

Figure 2.

Establishing the device response to changes in primer length. The interlocked $\alpha-\mathrm{HL} \cdot \mathrm{DNA}$ PEG transmembrane complexes containing primers of different lengths. (a) 23 nucleotides (+0), (b) 27 nucleotides (+4), (c) 29 nucleotides (+6), and (d) 31 nucleotides (+8). All primer sequences are fully complementary to the 3 '-end of the template sequence (Fig. 1). As the length of the primer increases, the ratio of PEG to DNA in the pore is increased (red pointers mark the PEG-DNA transition in each complex). (e) Primer length differences manifest themselves in the characteristic current-voltage $(I-V)$ trace of each complex. The largest and most reproducible current difference between the four complexes was observed at $+40 \mathrm{mV}$ (vertical dotted line). Currents were averaged from the final $0.2 \mathrm{sec}$ of the $2 \mathrm{sec}$ ion current recording at each potential. The $I-V$ traces were averaged from 10 or more recordings. Experiments were performed under conditions appropriate for DNA polymerase activity (150 $\mathrm{mM} \mathrm{KCl}, 25 \mathrm{mM}$ Tris, $4.5 \mathrm{mM} \mathrm{MgCl} 2$, at $\mathrm{pH} 8.0$ and $22 \pm 2{ }^{\circ} \mathrm{C}$, see Fig. S1. 


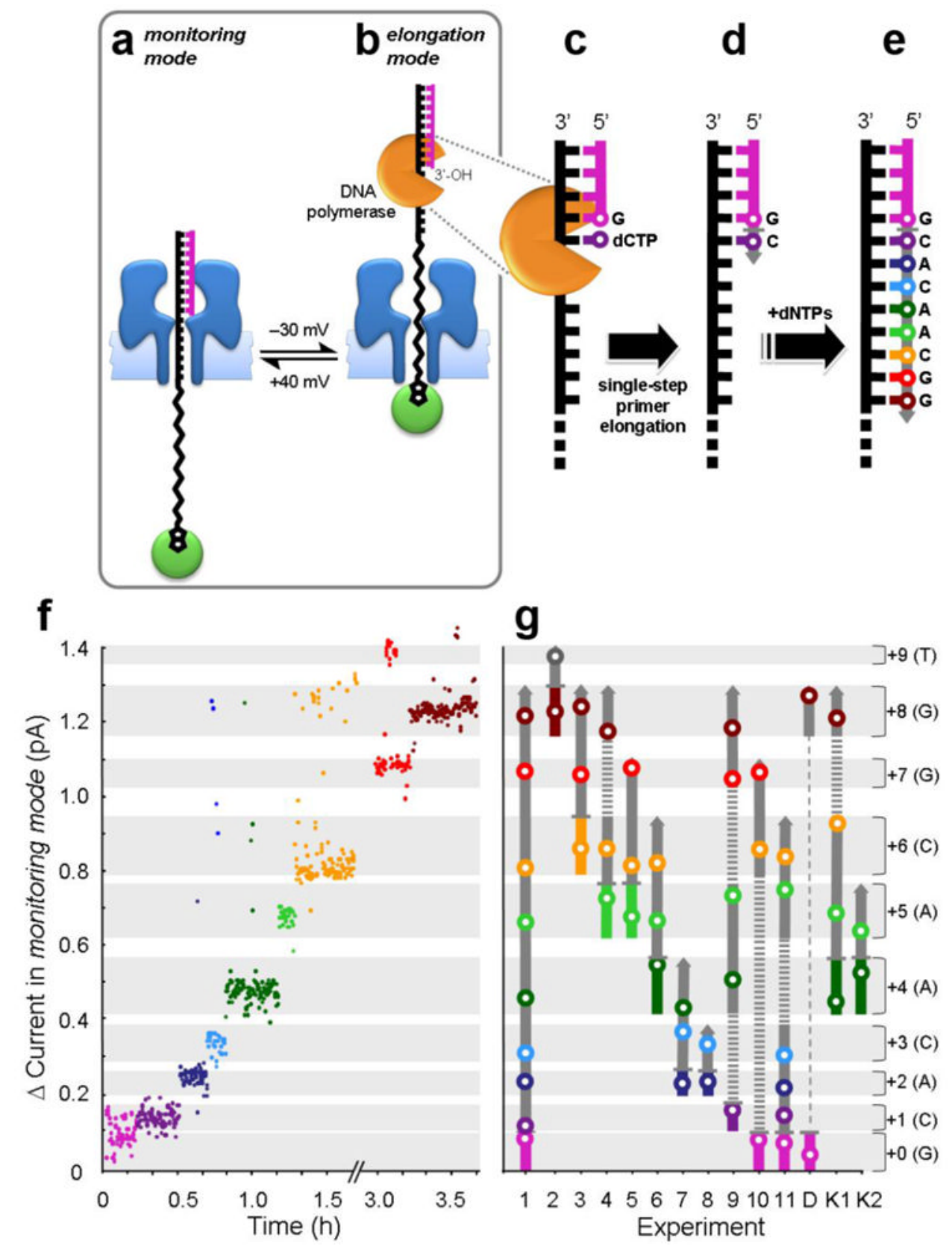

Figure 3.

Monitoring DNA polymerase-catalyzed single-nucleotide primer extensions. (a) The length of the DNA primer in a fully interlocked $\alpha-\mathrm{HL} \cdot \mathrm{DNA}-\mathrm{PEG}$ complex is reported by ion current measurements in the monitoring mode $(+40 \mathrm{mV})$. (b) In the elongation mode $(-30 \mathrm{mV})$, the 3'-OH of the primer is accessible to the DNA polymerase. (c) DNA polymerase bound to the starting primer-template complex, with the correct incoming deoxynucleotide triphosphate. (d) DNA polymerase catalyzes the elongation of the primer by incorporating a single dNTP against the DNA template threaded through the pore. (e) Templated extension of the primer sequence after multiple base incorporation steps. (f) A plot of cumulative change in the ion channel current recorded in the monitoring mode versus time. The data contains nine distinct current levels corresponding to the starting primer $(+0$, Fig. $2 \mathrm{~A})$, the seven intermediate polymerized states, and the extended primer (+8, Fig. 2d). Primer extension was paused for over one hour 
after the incorporation of 6 bases (+6) until the required dGTP was added (note the broken time axis). (g) Alignment of multiple experiments initiated with primers of different lengths (vertical colored bars). The dark gray arrows indicate the direction of primer extension. The white dots in colored circles represent the average cumulative ion current for each length of primer (s.e.m. $\pm 0.05 \mathrm{pA}$ ). The horizontal light gray stripes indicate the average current maxima and minima for each primer length. The numbers to the right indicate the base extension relative to the shortest primer $(+0)$, and the letters in parentheses identify the base at the 3 ' end of each primer. Dashed lines represent nucleotide incorporation steps that were not sampled by current recordings. Experiment 1 corresponds directly to the current recordings shown in Fig. 3F. Experiment $\mathrm{D}$ shows the displacement of a short primer $(+0)$ by a longer primer $(+8)$ as described in the main text. TopoTaq DNA polymerase was used in experiments 1 to 11 . The Klenow fragment (exo-) of E. coli DNA polymerase I was used in experiments K1 and K2. Polymerization was terminated with the appropriate dideoxynucleotide triphosphate (ddNTP) in experiments 2, 7, and K2. Experimental conditions were as described in Fig. 2, but with the addition of 2 nM DNA polymerase and 0.01-0.2 mM dNTPs. See Fig. S4-S17 for complete current versus time recordings for all experiments. 